\title{
Diagnosis and Analysis of Diagnosis Properties Using Discrete Event Dynamic Systems
}

\author{
Magnus Larsson \\ Department of Electrical Engineering \\ Linköping University, S-581 83 Linköping, Sweden \\ WWW: http://www.control.isy.liu.se \\ Email: magnusl@isy.liu.se
}

January 18, 1999

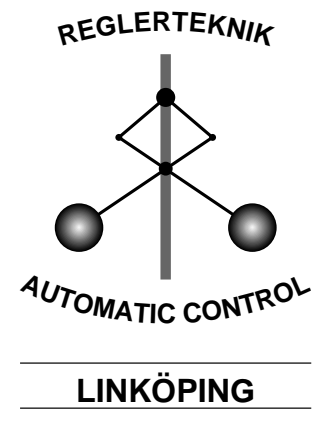

Report no.: LiTH-ISY-R-2093

Presented at CDC'98

Technical reports from the Automatic Control group in Linköping are available by anonymous ftp at the address ftp. control. isy.liu. se. This report is contained in the compressed postscript file 2093.ps. Z. 


\title{
Diagnosis and Analysis of Diagnosis Properties Using Discrete Event Dynamic Systems
}

\author{
M. Larsson \\ Department of Electrical Engineering \\ Linköping University \\ S-581 83 Linköping, Sweden \\ magnusl@isy.liu.se
}

\begin{abstract}
The basic motivation for the research presented in this article is the fact that things go wrong. With the growing complexity of todays engineering systems, the need has arisen for systematic approaches to failure diagnosis. This paper presents an approach for modeling and diagnosis of systems that fall in the area of discrete event dynamic systems.

We use a relational framework for discrete event dynamic systems focusing on a conceptually simple representation of the relationships between inputs, outputs and states of a discrete event system.

A fault is said to be detectable if there exists a transition in the system model that leads to a detection in a finite number of steps. The transition necessary for detection can automatically be computed from the system model under certain conditions. We also show how to compute the finest possible fault partition under a single fault assumption.
\end{abstract}

Keywords: discrete event, diagnosis, analysis

\section{Introduction}

The basic motivation for the topic of this article is that things go wrong. More specifically, in engineering systems things stop working or break, a valve gets stuck, a communication link breaks down, an engine gets overheated, etc.. These faults need to be timely and accurately detected and isolated for safety, reliability and availability of the system.
An approach for modeling and diagnosis of systems that fall in the area of discrete event dynamic systems (DEDS or DES) is proposed. We will with the term diagnosis mean fault detection and fault isolation, i.e., from observations of the system determine if a fault has occured, where it occured and what it is. The approach is applicable to systems that at some level of abstraction have an interesting discrete event dynamics that can display faulty behavior. The systems suitable for this approach typically consist of several interacting components where abrupt, but non-catastrophic, faults can occur in the components.

The work presented here is closely related to the formal language approach to diagnosis of discrete event systems by Sampath et al. [1] in that the same diagnosis problem is adressed. One of the main differences is that we must restrict ourselves to simpler, less powerful, models, but we are able to obtain more powerful analysis results.

\section{Modeling}

What we want is a conceptually simple representation of DEDS that is suitable for computer representation and computation and that captures the relevant properties. Our stab at this is to use variables for inputs, outputs and states of the system, and represent how the outputs and next states depend upon the inputs and current states with a relation. A relation is simply a mapping from a finite domain to the Boolean domain. The mapping takes the value true for the elements "included" in the relation. 
Now we give a general and very natural definition of a model, where the different parts will be further discussed in this section.

\section{Definition 2.1 DEDS}

A DEDS model of a (physical) system consists basically and very naturally of the three parts:

- State, input and output variables, usually denoted $x, u$ and $y$, with defined, discrete, value ranges.

- A relation (relational model) $M\left(x, u, y, x^{+}\right)$in the variables, stating exactly how to compute the outputs and next state from the inputs and current state.

- A real world interpretation of the variables, or rather of their different values.

\section{Example Two views of a DEDS}

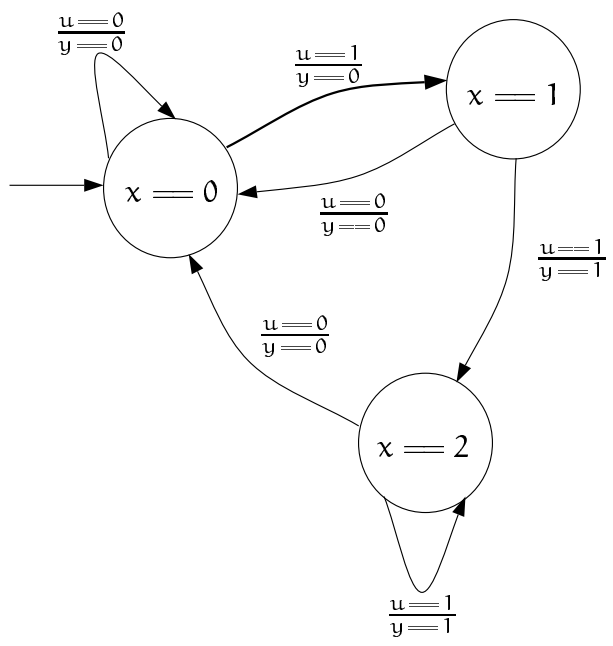

Figure 1: Finite state machine

A DEDS can be given graphically as a state machine as shown in Figure 1, or we can use equations and finite functions to describe how to compute the next states and outputs from the current state and inputs

$$
\left\{\begin{array}{r}
x^{+}=f(x, u) \\
y=g(x, u)
\end{array}\right.
$$

which also can be expressed using a relation

$M\left(x, u, y, x^{+}\right)=\left(x^{+}=f(x, u)\right) \wedge(y=g(x, u))$.

\section{Example: valve/pump sys- tem}

To illustrate the methods and concepts, we consider a very simple example system taken from [2], where it is treated in a formal language framework. The system is shown in figure 2 and consists of a valve, a pump, a sensor that indicates presence or absence of flow, and a controller. In the figure, the

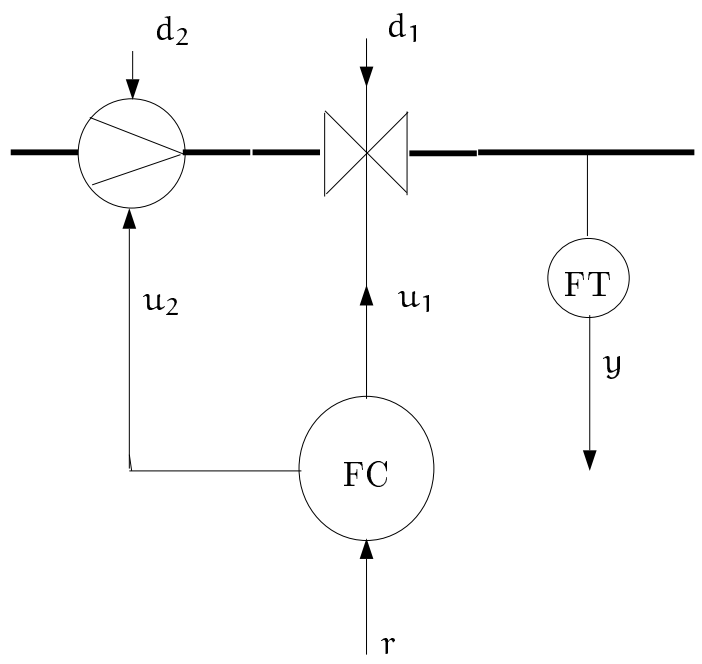

Figure 2: Flowchart of a simple system.

controller is denoted FC (Flow Control) and the sensor is denoted FT (Flow Transmission). The pump and the valve can be either on/off respectively open/closed. The valve and the pump can both fail in two different ways, stuck open/on or stuck closed/off. The goal is to build a model of this system that can be used to detect faults in the valve and the pump based on the sensor measurements, the known inputs and the known state of the controller.

If we for a moment assume that the pump cannot fail, it is for this simple system easy to see directly 
how to detect faults in the valve. If the valve is supposed to be open and the pump is on but we measure no flow, then the valve is stuck closed and vice versa for stuck open. It does not take many more faulting components and sensors to make the heuristic construction of such rules cumbersome, though.

One of the main features of this DEDS approach and the approach in [1], is the ability to analyse diagnosability properties. The analysis methods are treated in Sections 7 and 8 . There we will show that when using a reasonable controller for the above pump/valve system, we can never detect the valve stuck open, which is not obvious at first glance even for this simple example.

We will model the system in Figure 2 componentwise, but the only component that will require state variables is the controller, so the modeling is quite simple.

The faults that can occur in the valve and the pump will be modeled simply as unknown inputs, $d_{1}$ and $d_{2}$ (see Figure 2), that can take the values

$\mathrm{d}_{\mathrm{i}}=0 \quad$ The valve/pump is stuck closed/off.

$d_{i}=1$ The valve/pump is working, i.e., responding to $u_{i}$.

$\mathrm{d}_{\mathrm{i}}=2$ The valve/pump is stuck open/on.

Usually, we will have one fault variable for each component, and that fault variable models all faults that can occur in that component. We will therefore distinguish between a fault variable $\mathrm{d}_{\mathrm{i}}$ and $\mathrm{a}$ fault, e.g., $\mathrm{d}_{1}=0$. We could also let the fault variable $d_{i}$ be a state variable, which would give us more modeling power. See [3] for more on this.

The other variables indicated in Figure 2 will be Boolean valued, with the obvious interpretation. I.e., $u_{1}=$ true means the valve is ordered open, etc.

The controller should use the two outputs $u_{1}$ and $u_{2}$ to control the valve and the pump in such a manner that we get flow in the system when the input $r$ is true and so that the pump never run when the valve is closed, since that could cause physical damage to the pump. The controller in Figure 3, with a two-valued state variable $x$, fulfills this specification. Observe that the FSM is only a way to

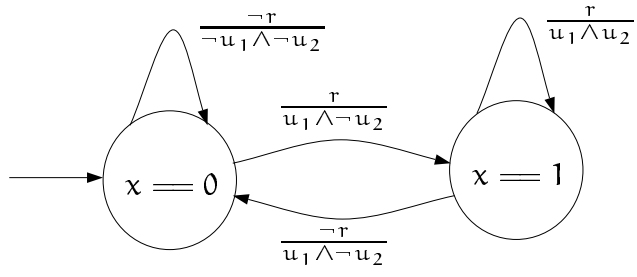

Figure 3: The Controller.

graphically visualize the table

\begin{tabular}{|c|c||l|l|l|}
\hline$r$ & $x$ & $u_{1}$ & $u_{2}$ & $x^{+}$ \\
\hline 1 & 0 & 1 & 0 & 1 \\
1 & 1 & 1 & 1 & 1 \\
0 & 1 & 1 & 0 & 0 \\
0 & 0 & 0 & 0 & 0 \\
\hline
\end{tabular}

We denote the corresponding relational model $C\left(x, r, u_{1}, u_{2}, x^{+}\right)$.

The controller used in [2] fulfills the same specification, but is modeled as a singleton finite automata.

The sensor measures if there is flow in the pipe. The condition for the sensor output $h$ to be true is that both the pump and the valve enables flow. This can be expressed as

where

$$
\begin{aligned}
& \phi_{\vee}\left(d_{1}, u_{1}\right)=\left(d_{1}=1 \wedge u_{1}\right) \vee\left(d_{1}=2\right) \\
& \phi_{P}\left(d_{2}, u_{2}\right)=\left(d_{2}=1 \wedge u_{2}\right) \vee\left(d_{2}=2\right)
\end{aligned}
$$

The sensor relation hence is

$$
\mathrm{S}\left(\mathrm{y}, \mathrm{u}_{1}, \mathrm{u}_{2}, \mathrm{~d}\right)=\left(\mathrm{y}=\left(\phi_{\mathrm{V}} \wedge \phi_{\mathrm{P}}\right)\right)
$$

We have implicitly assumed that flow occurs or is cut off instantaneously when the corresponding control signal is set, or rather that the real, physical system has reached steady state when the measurement is performed.

In general, the total model of a system is obtained by taking the synchrounous product of the submodels, see [3]. Since in this simple example, only one component is modeled with actual states, the total model of the system is obtained by

$$
\begin{aligned}
& M^{\prime}\left(x, d, r, u, y, x^{+}\right)= \\
& \quad S(y, u, d) \wedge C\left(x, r, u, x^{+}\right) .
\end{aligned}
$$


where $d=\left\{d_{1}, d_{2}\right\}$ are fault inputs, $x$ is a state and $r$ is an input. The variable $y$ is an output, and also $u=\left\{u_{1}, u_{2}\right\}$ has become outputs, or internal signals since they are given by the controller.

Since $u_{1}$ and $u_{2}$ are internal signals completely specified by the controller, i.e., by the input $r$ and state $x$, we we can simplify our relation by existential quantification without losing any information.

$$
M\left(x, d, r, y, x^{+}\right)=\exists u . M^{\prime}\left(x, d, r, u, y, x^{+}\right)
$$

I.e., we project the total behavior onto the external behavior only. This relation is the final model of the system.

\section{Diagnosis}

With diagnosis we mean fault detection and fault isolation, i.e., we wish to from observations of the system determine if a fault has occured, where it occured and what it is. This rather loose statement will be given a more strict interpretaion below.

Assume that a relational model of the system is given

$$
M\left(x, d, u, y, x^{+}, d^{+}\right)
$$

where the states $x$, fault variables $d$, inputs $u$ and outputs $y$ can be vectors of variables. Any of the fault variables can be either an input or a state. If the fault $d_{i}$ is an input, then $d_{i}^{+}$is not present in the relation (2). A fault variable will always have exactly one normal value, denoted $d_{i, N}$ and one or more fault values, denoted $d_{i, k}$.

In Sections 5 and 6 we discuss fault detection and fault isolation and analysis methods for certain detection and isolation properties given a relational model $M$ will be discussed in Sections 7 and 8 .

\section{$5 \quad$ Fault detection}

Fault detection is to determine as quickly as possible if something has gone wrong from knowledge of the system and observations. In our case, the knowledge is represented by the relational model (2), so this is an example of model based diagnosis.

A natural view to take is that if it is possible that the system operates normally, then it probably does so. In the example in Section 3 we would otherwise for instance warn that the valve may be stuck closed at every time step the valve is supposed to be closed.

\section{Definition 5.1 Detection}

Consider the relational model $M\left(x, d, u, y, x^{+}, d^{+}\right)$.

If at any time the known values of states, inputs and outputs together with $M$ are not consistent with all fault variables having their normal value, then a fault has been detected.

We will assume that we can observe when the system changes state. The only uncertainty, or nondeterminism, in the ordinary states $x$ that we allow when doing diagnosis, is induced by the unknown fault state variables. Also, for the analysis methods in Section 7, it is assumed that the fault variables do not influence the ordinary state at all, i.e., that all fault information is in the fault variables. To examine how important this rater harsh restriction is in practice and, possibly, how to remove it, will be a matter of further research.

Given the current state, current input and the observed output, the next state and possible values of the unknown fault variables can be calculated from the relational model.

$$
\begin{gathered}
E\left(d, y, x^{+}, d^{+}\right)=\exists x, u, y \cdot M\left(x, d, u, y, x^{+}, d^{+}\right) \wedge \\
K(x, d) \wedge u(u) \wedge Y(y)
\end{gathered}
$$

where the relations $K, U$ and $Y$ contains the information of the current state (remember, fault variables can be states), input and observed output respectively.

We can separate the possible values of the current fault variables, and the information on the next state.

$$
\begin{aligned}
\mathrm{F}(\mathrm{d}) & =\exists \mathrm{x}^{+}, \mathrm{d}^{+} . \mathrm{E}\left(\mathrm{d}, \mathrm{x}^{+}, \mathrm{d}^{+}\right) . \\
\mathrm{K}_{\text {new }}\left(\mathrm{x}^{+}, \mathrm{d}^{+}\right) & =\exists \mathrm{d} . \mathrm{E}\left(\mathrm{d}, \mathrm{x}^{+}, \mathrm{d}^{+}\right)
\end{aligned}
$$

When the controller changes state, we replace $K$ with $\mathrm{K}_{\text {new }}$.

A fault is then detected when the normal values of the fault variables are not among the values consistent with the observations. We let the values of the fault variables indicating normal behavior be represented by the constant relation $N(d)$. Fault detection is then carried out by checking

$$
F(d) \wedge N(d) \stackrel{?}{=} \text { false. }
$$


An interpretation of this detection procedure is to say that we simulate the model one time step for all possible faults, and then compare the predictions with the observations, ruling out the impossible values of the fault variables.

\section{Fault isolation}

Fault isolation takes place after fault detection, and is the process of determining where the detected fault has occured, and what type of fault it is. The "where" question is basically to determine which component has malfunctioned, or in other words to determine which fault variable has caused the fault detection.

All information we have available about the occured fault is in the relation $F(d)$, where $d=$ $\left\{d_{1}, \ldots, d_{n_{d}}\right\}$. Every solution to $F(d)$ is a list of values for $\left\{d_{1}, \ldots, d_{n_{d}}\right\}$ that explains the observed behavior. The solutions of $F(d)$ with the normal values $d_{i, N}$ removed will be called explanations and the set of all explanations will be called the explanation set.

The fault detection was triggered by the fact that there was no solution to $F(d)$ with only normal values for the fault variables. If $F(d)$ is empty, i.e., $F(d)=$ false, then the model cannot explain the behavior. Assume that $F$ is nonempty.

The explanation set can contain several explanations, many of which are supersets of other explanations. By removing every explanation that has another explanation as a subset, we get what we will call a minimal explanation set and this is the output of the fault isolation procedure.

To keep it as simple as possible, we can use that for many systems in practice it is very common that only one fault occur at a time. Under the assumption that only one fault can occur at a time, the minimal explanation set consists of singletons (single faults).

\section{Analysis of detection prop- erties}

We would like to analyze if and when we can detect a certain fault. The very simple idea is to pick out the behavior for which the fault is not detected. The analysis is carried out for each fault value of each fault variable separately under a single fault assumtion. That is, we analyse detectability properties under the assumption that only one fault occurs at a time, with the rest of the fault variables set to have their normal value. We also assume that the fault do not influence the "ordinary" state evolution of the system. To establish notation, let the fault under consideration be $\mathrm{d}_{\mathrm{i}}=d_{i, k}$ and let $M_{i}\left(x, d_{i}, u, y, x^{+}, d_{i}^{+}\right)$be the system model with all other fault variables set to their normal value.

\section{Definition 7.1 Detectable fault}

A fault $d_{i, k}$ is said to be detectable if there exists a controllable transition, i.e., values of $x$ and $u$, in the system model under the single fault assumption that leads to a detection in a finite number of steps.

The transition that leads to a detection corresponds to the indicator event in the formal language approach by Sampath et al. [1]. We also say that $M$ is detectable if all faults are detectable. This definition of detectability would then correspond to I-diagnosability [1]. Note that detectable fault and detectable are properties of the relational model $M$ and not of the real system.

In the following, algorithmic methods will be presented both to decide if a fault is detectable, and to decide which transitions and hence which inputs that are necessary to detect the fault.

\subsection{Ambivalent behavior}

We will say that the fault detection procedure is ambivalent w.r.t. $d_{i, k}$ when the normal value $d_{i, N}$ and the fault value $d_{i, k}$ of the fault variable $d_{i}$ is in the estimation set $F(d)$ (4) simultaneously, i.e., when the fault and the normal behavior is consistent with the observations. With our method of modeling, with a fault as an unobservable input or a nondeterministic state variable, at least one possible fault is bound to be in the estimation set at almost all times. E.g., in the valve/pump example in Section 3 we have that when the valve is closed there is always the possibility that it is stuck closed and we cannot know without trying to open the valve.

The state and input combinations for which the fault detection procedure is ambivalent w.r.t. $d_{i, k}$ 
can be computed as

$$
A_{d_{i, k}}(x, u, y)=\exists x^{+}, d_{i}^{+} \cdot M_{i}\left[d_{i} \mapsto d_{i, N}\right] \wedge M_{i}\left[d_{i} \mapsto d_{i, k}\right] \quad \tilde{c}_{d_{i, k}}\left(x, u, x^{+}\right)=\exists y \cdot c_{d_{i, k}}\left(x, u, y, x^{+}\right)
$$

where $A_{d_{i, k}}$ stands for ambivalent w.r.t. the fault $d_{i, k}$. Note that we consider the ambivalence for each state/input combination, i.e., each transition, separately, see Figure 4 . This is possible since all fault information is carried by $d_{i}$. The reason that we can quantify w.r.t. $x^{+}$and $d_{i}^{+}$in (7) is that neither are used as observations when computing the estimation set (3).

We would also like to know if it is possible to have a sequence of inputs and states such that we never can detect $d_{i, k}$, i.e., we want to find the loops in $M_{i}\left(x, d_{i}, u, y, x^{+}, d_{i}^{+}\right)$where the fault detection procedure is ambivalent w.r.t. $d_{i, k}$. These loops will be called the infinitely ambivalent behavior.

To find this behavior, we restrict $M_{i}$ to the ambivalent behavior given by $A_{d_{i, k}}$. The resulting relational model will be denoted $c_{d_{i, k}}^{\prime}\left(x, u, y, x^{+}\right)$and it contains exactly the behavior for which $d_{i, k}$ cannot be detected.

$$
\begin{aligned}
& c_{d_{i, k}}^{\prime}\left(x, u, y, x^{+}\right)= \\
& \exists d_{i}, d_{i}^{+} \cdot M_{i}\left(x, d_{i}, u, y, x^{+}, d_{i}^{+}\right) \wedge A_{d_{i, k}}(x, u, y) .
\end{aligned}
$$

We then find the infinitely ambivalent behavior by finding the loops in $\mathrm{c}_{d_{i, k}}^{\prime}$ and denote the resulting relational model $c_{d_{i, k}}\left(x, u, y, x^{+}\right)$. For details on how the loop detection is performed, see [3, 4].

The methodology is visualised in Figure 4.

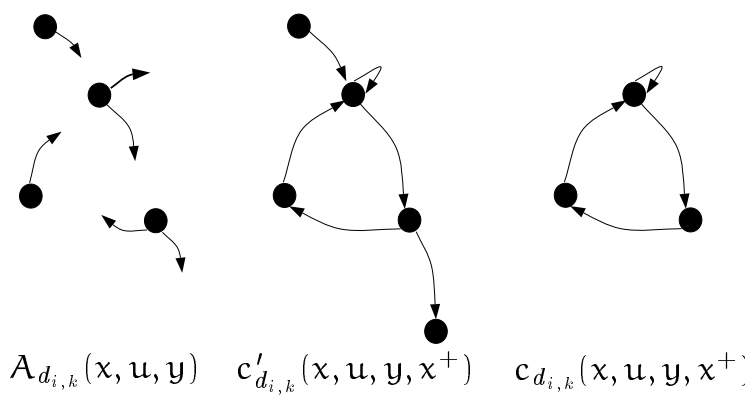

Figure 4: Possible scenario for the detectability analysis process.

To generate a sample path of $\mathrm{c}_{d_{i, k}}$, just do random simulation or, if it is feasible, visualize as a FSM (for an example, se Section 9).

\subsection{Detectability test}

The faults that never can be detected regardless of input are called nondetectable according to Definition 7.1 and are of course of special interest. A procedure for finding out if a fault is nondetectable will now be presented.

To automatically check for detectability, we need the transitions possible to force on the the system when $\mathrm{d}=d_{i, k}$ or $\mathrm{d}=d_{i, N}$ and that possibly leads out of $c_{d_{i, k}}\left(x, u, y, x^{+}\right)$.

$$
\begin{aligned}
& \tilde{M}_{d_{i, k}}\left(x, u, x^{+}\right)=\exists d_{i}, y, d_{i}^{+} \cdot M_{i} \wedge \\
& \left(d_{i}=d_{i, N} \vee d_{i}=d_{i, k}\right) \wedge \text { Project } x . \tilde{c}_{d_{i, k}}
\end{aligned}
$$

For a relation $\mathrm{R}\left(z_{1}, z_{2}\right)$, the Project $\cdot$. operator is defined as Project $z_{1} \cdot R=\exists z_{2}$. R.

Note that we do not restrict the next states $x^{+}$ of $\tilde{M}_{d_{i, k}}$ to the states present in $c_{d_{i, k}}$, since that could remove transitions leading out of $c_{d_{i, k}}$. The relational model $\tilde{M}_{d_{i, k}}$ will be called the possible, or forcible, behavior of $M$ w.r.t. $d_{i, k}$.

The fault is then detectable iff there exists transitions in $\tilde{M}_{d_{i, k}}\left(x, u, \chi^{+}\right)$that are not present in $\tilde{\mathbf{c}}_{d_{i, k}}\left(x, u, x^{+}\right)$and hence will lead to a detection. The fault $d_{i, k}$ is then nondetectable iff the test

$$
\tilde{\mathbf{c}}_{d_{i, k}} \stackrel{?}{=} \tilde{M}_{d_{i, k}}
$$

comes out true. That $\tilde{\mathbf{c}}_{d_{i, k}} \subseteq \tilde{M}_{d_{i, k}}$ is clear from construction.

The transitions that lead out of $\tilde{\mathbf{c}}_{d_{i, k}}$, and hence will lead to a detection in a finite number of steps are explicitly given by the relation

$$
\neg \tilde{M}_{d_{i, k}}\left(x, u, x^{+}\right) \wedge \tilde{c}_{d_{i, k}}\left(x, u, x^{+}\right)
$$

The so found transitions then correspond to the indicator events in the formal language approach $[1]$.

\section{Analysis of isolation proper- ties}

The question to answer is, which pair of faults cannot be distinguished (isolated)? We make the following definition. 
Definition 8.1 Isolatable faults

For the relational model $M\left(x, u, y, x^{+}, d^{+}\right)$, we will call the two faults $d_{i, k}$ and $d_{j, l}$ isolatable if there exists a controllable transition, i.e., values of $x$ and $u$, in $M$ which gives different behavior, i.e., outputs or next states, for the two faults. All fault variables $d_{s}$ are assumed to have their normal value $d_{s, N}$ for $s \neq i, j$.

The property in Definition 8.1 can be checked as follows. Calculate the two relational models $\mathrm{b}_{d_{i, k}}$ and $\mathrm{b}_{d_{j, l}}$.

$$
\begin{aligned}
\mathrm{b}_{d_{i, k}}\left(x, \mathrm{u}, \mathrm{y}, \mathrm{x}^{+}\right) & =\mathrm{M}_{\mathrm{i}}\left[\mathrm{d}_{\mathrm{i}} \mapsto d_{i, k}, \mathrm{~d}_{\mathrm{i}}^{+} \mapsto d_{i, k}\right] \\
\mathrm{b}_{d_{j, l}}\left(\mathrm{x}, \mathrm{u}, \mathrm{y}, \mathrm{x}^{+}\right) & =\mathrm{M}_{\mathrm{j}}\left[\mathrm{d}_{\mathrm{j}} \mapsto d_{j, l}, \mathrm{~d}_{\mathrm{j}}^{+} \mapsto d_{j, l}\right]
\end{aligned}
$$

The relational model $b_{d_{i, k}}\left(x, u, y, x^{+}\right)$represents the behavior of the system, under the single fault assumption, if the fault $d_{i, k}$ has occured. If $\mathrm{b}_{d_{i, k}}=$ $\mathrm{b}_{d_{j, l},}$, i.e., the system has exactly the same behavior for both faults, then the errors $d_{i, k}$ and $d_{j, l}$, cannot be distinguished for any input/state sequence.

To find all pairs of faults that cannot be distinguished, we have to perform all possible pairwise comparisons. Since the analysis is done off line, this should be reasonable. What we get is a list of pairs of faults that cannot be isolated without, e.g., changing the controller or adding sensors.

The finest possible fault partition, under the single fault assumption, for which the faults in different partitions still are distinguishable, then is given by partitioning the faults with identical behavior together.

\section{Valve/pump example revis- ited}

We will now analyse the valve/pump system modeled in Section 3 with the methods discussed in this section.

We will use the notation $d_{1, N}=1, d_{1,0}=0$ and $d_{1,2}=2$ for valve normal, stuck closed and stuck open respectively. Analogous for the pump.

It turns out that the possible transitions obtained as (10), $\tilde{M}_{d_{1,0}}$, etc. are the same for all four faults, and the corresponding FSM is shown in Figure 5.

The relational models for the ambivalent behavior $\tilde{\mathbf{c}}_{d_{1,0}}, \tilde{\mathbf{c}}_{d_{1,2}}, \tilde{\mathrm{c}}_{d_{2,0}}$ and $\tilde{\mathbf{c}}_{d_{2,2}}$ obtained by the analysis procedure outlined by equations (7) to (11), are visualised in figure 6 .

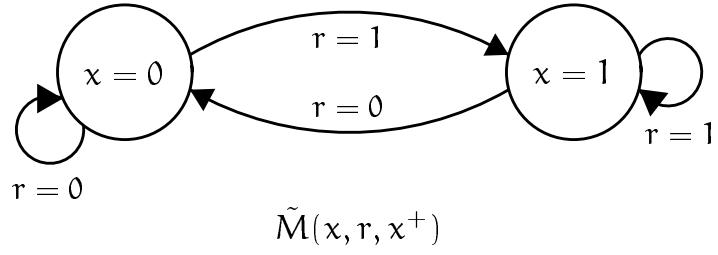

Figure 5: The possible transitions for the system.

If we, e.g., compare the ambivalent behavior $\tilde{\mathbf{c}}_{d_{0}}\left(x, \mathrm{r}, \mathrm{x}^{+}\right)$in Figure $6(\mathrm{a})$ and the possible behavior $\tilde{M}_{d_{0}}\left(x, r, x^{+}\right)$in Figure 5 , we see that $d_{1,0}$ is indeed detectable. The transition for $x=1, r=1$ in figure 5 can be forced on the system, but is not present in figure $6(\mathrm{a})$ and hence leads out of $\tilde{\mathrm{c}}_{d_{1,0}}$.

If we assume that the pump is working correctly, this means that if flow is ordered, $r=1$, the valve is supposed to open and the pump starts pumping and we should get flow. If the valve is stuck closed, though, we do not get any flow and the fault $d_{1,0}$ can be detected.

If we instead compare the ambivalent behavior $\tilde{c}_{d_{1,2}}\left(x, r, x^{+}\right)$in Figure 6(b) with the possible behavior $\tilde{M}\left(x, r, x^{+}\right)$, we see that the fault $d_{1,2}$ is nondetectable according to the test (11), since $\tilde{\mathrm{c}}_{d_{1,2}}=\tilde{M}$.

The explanation is that to detect the valve stuck open by flow measurements, we would have to run the pump when the valve is supposed to be closed, and for safety reasons we, i.e., the controller, never do that.

Following the same line of reasoning, it is clear from Figures 6(c) and 6(d) that also pump stuck on $\left(d_{2,0}\right)$ and pump stuck off $\left(d_{2,2}\right)$ are detectable.

When we follow the procedure outlined in Section 8 to decide wich faults are pairwise isolatable, it turns out that $d_{1,0}$ and $d_{2,0}$, i.e., valve stuck closed and pump stuck off, cannot be distinguished. In either case, we simply do not get any flow at the flow sensor.

Hence, if we introduce the fault partition

$$
\left\{\left\{d_{1,0}, d_{2,0}\right\},\left\{d_{1,2}\right\},\left\{d_{2,2}\right\}\right\}
$$

these groups of faults are distinguishable from each other, apart from that $d_{1,2}$ is undetectable. 


\section{Conclusions}

We have proposed an approach for modeling and diagnosis of systems that fall in the area of discrete event dynamic systems and shown how to perform analysis of diagnosability properties. We have presented automatic procedures for finding which faults are detectable, to find the necessary behavior to detect a fault and to find a minimal fault partition.

\section{References}

[1] M. Sampath, R. Sengupta, S. Lafortune, K. Sinnamohideen, and D. Teneketzis. Diagnosability of discrete-event systems. IEEE Transactions on Automatic Control, 40(9):1555-1575, September 1995.

[2] M. Sampath, R. Sengupta, S. Lafortune, K. Sinnamohideen, and D. Tenektzis. Failure diagnosis using discrete event models. Technical Report CGR-94-03, Department of Electrical Engineering and Computer Science, The University of Michigan, Ann Arbor, USA, May 1994.

[3] M. Larsson. On Modeling and Diagnosis of Discrete Event Dynamic Systems. Licentiate thesis LIU-TEK-LIC-1997:49, Department of Electrical Engineering, Linköping University, Linköping, Sweden, October 1997.

[4] J. Gunnarsson. Symbolic Methods and Tools for Discrete Event Dynamic Systems. Phd thesis 477, Department of Electrical Engineering, Linköping University, Linköping, Sweden, May 1997.

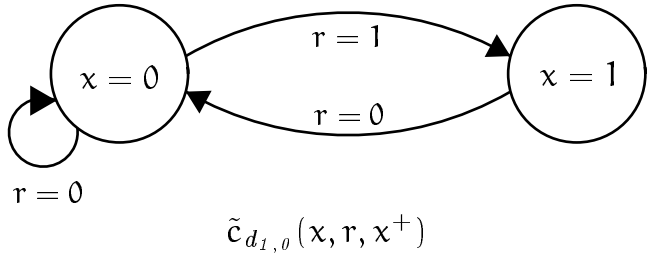

(a) The transitions for which $d_{1,0}$ (valve stuck closed) cannot be detected.

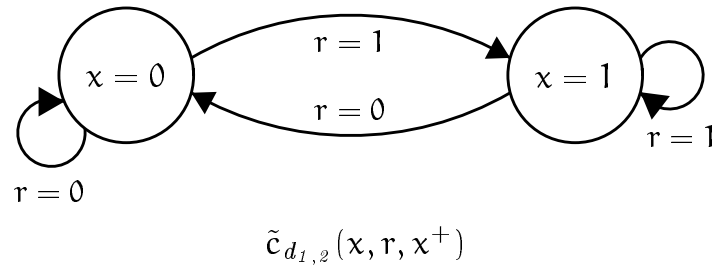

(b) The transitions for which $d_{1,2}$ (valve stuck open) cannot be detected.

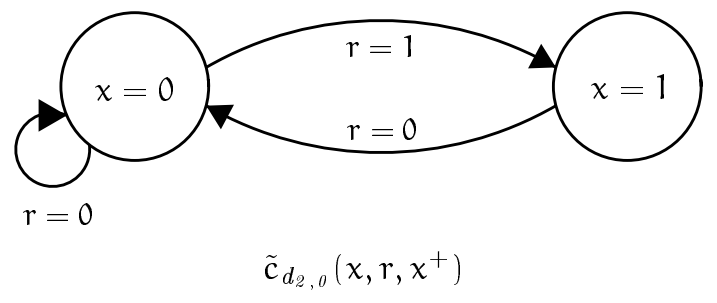

(c) The transitions for which $d_{2, O}$ (pump stuck off) cannot be detected.
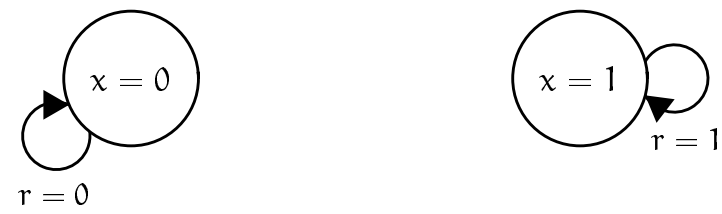

$$
\tilde{\mathbf{c}}_{d_{2,2}}\left(x, r, x^{+}\right)
$$

(d) The transitions for which $d_{2,2}$ (pump stuck on) cannot be detected.

Figure 6: The ambivalent behavior w.r.t. $d_{1,0}, d_{1,2}, d_{2,0}$ and $d_{2,2}$. 